\title{
Avaliação de jogos digitais educativos: considerações e conclusões de um levantamento bibliográfico
}

\author{
Isa de Jesus Coutinho, PPGEduc/UNEB, isacoutinho13@hotmail.com \\ Lynn Rosalina Gama Alves, PPGEduc/UNEB, SENAI/CIMATEC. \\ lynnalves@gmail.com
}

\section{Resumo}

Este artigo, que é parte do doutorado de uma das autoras, teve como objetivo socializar os resultados de um levantamento bibliográfico sobre as produções referentes avaliação de jogos digitais educativos no Brasil e nos países estrangeiros apontando considerações e conclusões, bem como possibilidades e limitações. Os principais resultados revelam que se trata de um conhecimento em construção com o maior interesse da área de computação para investigação do tema e com amplo investimento em avaliações formativas, ou seja, com o jogo em desenvolvimento. Como conclusão recomenda-se a ampliação deste campo de pesquisa nos cenários brasileiros, em particular na área da educação.

Palavras chave: jogos digitais, avaliação de jogos digitais, avaliação da qualidade de jogos digitais, jogos digitais e aprendizagem.

\begin{abstract}
This article, which is part of the doctorate of one of the authors, aimed to socialize the results of a bibliographic survey on the productions related to the evaluation of digital educational games in Brazil and in foreign countries, pointing out considerations and conclusions, as well as possibilities and limitations. The main results reveal that this is a knowledge in construction with the greatest interest of the area of computation to investigate the subject and with a wide investment in formative assessments, that is, with the game in development. As conclusion it is recommended to expand this field of research in Brazilian settings, particularly in the area of education.
\end{abstract}

Keywords: digital games, digital games assessment, evaluation of the quality of digital games, digital games and learning.

\section{Introdução}

A produção de games tem se destacado como o terceiro maior faturamento mundial, ultrapassando o cinema e a música juntos. Fazendo um recorte para a indústria brasileira $^{a}$ no ano de 2013 foram desenvolvidos 1417 jogos, sendo quase a metade deles, $621(43,8 \%)$, voltados para a educação. Paralelamente a isso, estudiosos ${ }^{\mathrm{b}}$ de diversas partes do mundo têm se dedicado a conhecer e avaliar o papel deste fenômeno cultural e suas contribuições para os processos de ensino e aprendizagem, além de sua eficácia como elemento educativo.

Neste emergente cenário, no qual a necessidade do reconhecimento da efetividade da aprendizagem baseada em jogos digitais é crescente, pesquisadores interessados no desenvolvimento de parâmetros de análise da experiência do jogar, e suas contribuições 
para os cenários educativos, têm configurado processos avaliativos a partir de distintas concepções teóricas, metodológicas e conceituais.

Este artigo tem como objetivo socializar os resultados de um levantamento bibliográfico ${ }^{c}$ sobre as produções referentes à avaliação de jogos digitais educativos no Brasil e nos países estrangeiros, apontando considerações e conclusões, bem como possibilidades e limitações. O interesse não é esgotar o tema nem dar a palavra final sobre as produções existentes, mas apresentar uma amostragem sobre o estado em que este tipo de conhecimento tem sido desenvolvido e aplicado.

\section{Metodologia}

Trata-se de um estudo de abordagem qualitativa, desenvolvido através de um levantamento bibliográfico, que envolveu as produções desenvolvidas nos últimos dez anos, tanto no Brasil quanto no exterior, sobre a avaliação de jogos digitais educativos. Duas questões norteadoras foram definidas no sentido de orientar os critérios de busca: ${ }^{d}$ a primeira visou identificar os estudos que apresentaram perspectivas de avaliação através de modelos, parâmetros roteiros e instrumentos; a segunda teve como objetivo apontar as pesquisas que intencionaram avaliar a qualidade dos jogos.

A seleção dos descritores de busca atendeu a seguinte organização: avaliação de jogos digitais com as combinações para jogos eletrônicos, games, videogames e videojuegos; avaliação da qualidade de jogos digitais, avaliação da qualidade de jogos digitais educativos, seguindo a combinação anterior; games quality evaluation; game analysis; evaluation of the quality of educational digital games.

Os critérios para inclusão dos estudos consistiram nas publicações entre os períodos de 2006 a 2015, com a redação dos idiomas Inglês, Espanhol e Português. Foram descartadas publicações como relatórios, resenhas, bibliografias e editoriais, assim como short papers.

O processo de busca automática, por meio da criação de filtros e estratégias de recuperação, ocorreu nas bases de dados do Simpósio Brasileiro de Jogos e Entretenimento Digital - SBGAMES; Seminário de Jogos Eletrônicos, Educação e Comunicação - SJEEC; Banco de Teses da Comissão de Aperfeiçoamento de Pessoal do Nível Superior - CAPES; The International Journal of Computer Game Research Games Studies; Elsevier; Google acadêmico; Scientific Electronic Library Online Scielo e em buscas aleatórias. A descrição e identificação das investigações obedeceram aos critérios das publicações ano a ano, tendo como requisitos a frequências das publicações.

Dois requisitos foram ponderados para a opção das fontes acima. O primeiro deles diz respeito à especificidade da fonte para o tema, como os exemplos do SBGAMES, SJEEC e o Games Studies que concentram seu investimento nos estudos dos jogos digitais. Já o segundo atendeu ao potencial de representatividade e relevância como espaço de busca por parte de pesquisadores. Destacaram-se o Banco de Teses da Capes, o Scielo, o Elsevier e o Google acadêmico.

A descrição e identificação das investigações obedeceram aos critérios das publicações ano a ano, tendo como requisitos a prevalência das frequências. De início, a mineração foi realizada considerando a primeira questão que tratou de identificar quais estudos propunham perspectivas para avaliar os jogos digitais voltados ao cenário educativo; em seguida, quais deles especificamente tinham interesse em avaliar a qualidade dos jogos. 
Por se tratar de um assunto abrangente e em expansão, a temática de avaliação dos jogos digitais envolve várias áreas de conhecimento não restrito ao campo da educação. $\mathrm{Na}$ amostragem aqui apresentada, pelo fato desta pesquisa ter como escopo a avaliação de jogos digitais com finalidade educativa, a maior concentração das observações e análises será investida nos estudos agregados à área educacional.

\section{Estudos brasileiros}

Foram contabilizados 30 trabalhos no Brasil. O SBGAMES, com 11 artigos, e o Google Acadêmico, com 10, foram as fontes nas quais se obteve o maior número de publicações relacionadas ao tema. Em seguida, o Banco de Teses da Capes, com 8 investigações, sendo 3 teses e 5 dissertações. Por fim, o SJEEC, com apenas um artigo. Não foram identificados estudos sobre o tema na base de dados do Scielo.

Ao analisar os Anais do SBGAMES, foi possível distribuir as publicações em três grupos: o primeiro grupo com intenção de identificar, através de processos avaliativos, critérios de bons jogos digitais para as práticas educativas, como os exemplos de (Tsuda et al. 2015), com o interesse em discutir os processos de avaliação dos jogos digitais e análises de métodos;(Petry et al. 2013), que se propuseram a explorar parâmetros, estratégias e técnicas de análise de jogo, além de (Leite e Mendonça, 2013), com a sugestão de diretrizes para o game design de jogos educacionais.

No segundo grupo, estavam integradas as produções cujo objetivo destinou-se a avaliar a eficácia da utilização dos jogos digitais para aprendizagem, a partir da proposição de Frameworks e Taxonomias, a exemplo de (Dourado et al. 2015; Júnior e Menezes 2015; Victal e Menezes 2015 e Sena et al. 2015), respectivamente. Ainda nesse grupo, a avaliação por perspectivas, trazida por (Dias et al. 2013), e pela satisfação do aprendiz, desenvolvida por (Rocha ,2015). Nestes estudos utilizou-se como dispositivo investigativo tanto questionários quanto a avaliação pelo próprio sistema do jogo através de softwares embarcados.

No terceiro grupo, identificaram-se os trabalhos cujo investimento propunha modelos da avaliação da usabilidade, com o destaque para as avaliações heurísticas, com a intenção de orientar o design dos games. Respectivamente (Gurgel et al. 2006; Mori et al. 2008; Silva e Joseli ,2015; Dias et al. 2015). Este grupo de artigos explicita um processo de avaliação formativa, ou seja, com o jogo ainda em desenvolvimento.

A busca no Google Acadêmico revelou mais da metade dos trabalhos, sete deles com o objetivo de propor modelos de avaliação para os jogos digitais com finalidade educativa. Neste contexto, ressaltam-se as investigações de (Savi et al. 2010; Medeiros e Schimiguel, 2012; Valle et al. 2013; Oliveira et al. 2015; Neves et al. 2014; Veridiano 2014; e Vilarinho e Leite 2015). Na sequência, os estudos de (Aguiar, 2010; França e Tedesco 2015) que apresentam como contribuição a avaliação para a melhoria do design do jogo educativo; e (Grimm e Calomeno 2009), com o objetivo de propor uma análise para orientar a utilização dos jogos eletrônicos educativos para a prática pedagógica.

As teses e dissertações datam a partir do ano de 2010. A primeira delas, desenvolvida através da dissertação de (Aguiar, 2010), resultou em um instrumento de avaliação de jogos eletrônicos educativos com o objetivo de buscar diretrizes para aperfeiçoar o processo de design. Na sequência, a tese de (Savi, 2011), com a proposta de um modelo para a avaliação da qualidade dos jogos. No ano seguinte, a dissertação de (Azevedo, 2012) propôs um roteiro para a análise pedagógica dos jogos digitais. A dissertação de (Araújo, 2013) apresentou um framework conceitual para apoiar a 
instrumentação de avaliação formativa da aprendizagem em jogos digitais; no mesmo ano, a dissertação de Ribeiro apresenta uma proposta de avaliação didático-pedagógica e ergonômica para jogos educacionais digitais voltados à língua portuguesa.

A tese de (Jappur, 2014) traz a proposição de um modelo conceitual para criação, aplicação e avaliação de jogos educativos digitais. Ainda naquele ano, o trabalho de (Rodrigues, 2014) propôs um instrumento de avaliação de jogos eletrônicos educativos do ensino fundamental 1. Por fim, (Dias, 2015), que, através de sua tese, desenvolveu um estudo que discute procedimentos analíticos para avaliação de jogos educacionais digitais.

No SJEEC apenas uma publicação foi localizada.Essa publicação destinou-se a estabelecer de forma crítica uma avaliação heurística como método potencial para avaliar a eficiência de um jogo educativo em transmitir seu conteúdo pedagógico. Este estudo desenvolvido por (Alves et al. 2013) objetivou discorrer sobre a avaliação heurística enquanto método utilizado para analisar softwares e sua possibilidade de adaptação para uso em jogos educacionais digitais.

\section{Estudos estrangeiros}

Considerando o escopo deste artigo, que não se destina a investigar o estado do conhecimento, mas apresentar um levantamento amostral do que foi produzido até o momento sobre o tema de interesse, deu-se continuidade à orientação de busca a partir das duas questões já definidas anteriormente, com o cuidado de destacar as produções que mais se aproximaram do interesse investigado, o que, de certa forma, não constituirá um quantitativo absoluto das produções encontradas, e sim uma amostragem sobre o cenário internacional. Foram destacados 24 trabalhos na Elsevier e apenas um no Game Studies. Neste último, o resultado da busca identificou apenas uma publicação relativa ao tema. Este referido estudo, desenvolvido por Consalvo e Dunton (2006), não traz como referência a avaliação de jogos digitais educativos. Seu foco de interesse relaciona-se a uma proposição genérica de avaliação para qualquer tipo de jogo.

Dos 24 estudos levantados, 16 descreveram modelos e frameworks: (Sweetser e Wyeth, 2005; Sandvik 2006; De Freitas e Oliver 2006; Leacock e Nesbit 2007; Hong et al. 2009; Fu et al. 2009; Pourabdollahian, et al. 2010; Nacke et al.2010; Serrano et al. 2012; Mohamed e Jaafar, 2010,2013; Mitgutsch e Alvarado 2013; Ahmad et al. 2014; Gee et al. 2014; Lepe-Salazar 2015; Kiili et al. 2016).

Para além deles, também foram encontradas publicações que apresentaram questionários como mecanismos de avaliação (Brockmyer et al. 2009); métodos (Sandvik 2006); Escala, (Wiebe 2014); instrumentos (Shchiglik, et al. 2008); Pinto et al. 2012).

Com relação aos estudos de revisão sistemática, foi possível destacar as contribuições de (Belloti et al. 2013; All et al. 2014; Marciano et al. 2014); Lukman et al. 2014), com o objetivo de identificar, estudar e analisar os processos de avaliação da eficiência e eficácia dos jogos digitais para aprendizagem descritos na literatura. Os resultados encontrados revelaram que os jogos digitais com finalidade educativa são compreendidos como jogos sérios (serious games), na medida em que sua finalidade não seja o entretenimento. Detectaram, também, que a complexidade metodológica em se definir um tipo específico de avaliação que evidencie a eficácia e eficiência dos jogos digitais para o ensino e aprendizagem gera uma variedade de desenhos metodológicos (All et al.2014), muitos deles sem critérios de análises e revisões definidos.

Ainda sobre as revisões, foram apontadas, pelos autores, a importância atribuída à medição do desempenho do jogador com relação ao jogo e, ao mesmo tempo, a avaliação 
das competências adquiridas (Belloti et al., 2013); por fim, a preocupação com o design do software e com a experiência do usuário (Marciano et al. 2014). Em resumo, os dados revelados pelas revisões foram unânimes em reconhecer a complexidade avaliativa dos jogos digitais tanto por sua natureza híbrida e multifacetada quanto pelo objetivo que é proposto em cada avaliação, isto é, o que se considera como importante a ser avaliado. Ademais, a insuficiência de estratégias avaliativas que evidenciem a eficiência e eficácia dos jogos digitais para o ensino e aprendizagem com revelações pontuais e genéricas sem resultados conclusivos.

Outro fato também observado foi um certo interesse por uma avaliação baseada no engajamento entre jogador e jogo como um aspecto importante para o ensino e aprendizagem. Os estudos apresentados por (Pourabdollahian et al. 2010; Wiebe, 2014; Brockmyer et al. 2009) integraram este contexto. Sweetser e Wyeth (2005); (Fu et al. 2009), assim como (Kiili et al. 2016), seguiram o mesmo percurso, subsidiados pela teoria do fluxo de Csikszentmihalyi, (2002).

E, mais ainda, o investimento em estratégias de avaliação, tendo como aspecto central o design do jogo, ou seja, a partir de sua concepção, esteve presente na maior parte das publicações, o que parece apontar para a necessidade de avaliar o jogo desde o seu processo inicial de desenvolvimento. Tal fato parece denunciar, assim, uma predominância pelos processos avaliativos formativos.

Com relação à área de concentração, as publicações relativas à área de Computação contabilizaram 12 trabalhos; a área de Educação, 10 e, em menor número, 2 estudos relativos à Psicologia.

\section{Considerações sobre os estudos brasileiros com relação aos internacionais.}

As investigações que tratam da avaliação dos jogos digitais educativos encontramse em constante expansão. No Brasil o aumento das publicações sugere importante crescimento a partir do ano de 2010, atingindo a maior frequência em 2015, quando se totalizam onze estudos após este período. Dos 30 trabalhos apreciados, a área de computação, com enfoque para o design de jogos, atingiu a maior proporção com $60 \%$ das publicações. Em educação, obteve-se $26,6 \%$ e, em menor proporção, outras áreas do conhecimento, totalizando $13,4 \%$.

Não foram observadas diferenças significativas com relação ao nexo investigativo e propositivo nas pesquisas entre os estudos nacionais e internacionais. Com o objetivo em propor perspectivas de avaliação como roteiros, modelos, frameworks, ambos se aproximam. O interesse em um processo de avaliação formativa (com o jogo em desenvolvimento) também foi frequente entre eles. Com relação aos aspectos avaliados, os estudos nacionais revelaram maior preocupação com os aspectos pedagógicos; já os internacionais, com a análise do processo de imersão, interação e engajamento, além da melhoria do conhecimento.

Outro aspecto que se evidenciou como diferencial diz respeito às metodologias de pesquisa. Os estudos estrangeiros se destacaram pela predominância de revisões sistemáticas com o envolvimento de um número importante de publicações, pela considerável amostragem de participantes nas pesquisas e pelo interesse nas metodologias experimentais. Já as pesquisas qualitativas se sobressaem nos estudos nacionais. Embora com um número menor de sujeitos neste tipo de pesquisa, foi possível observar que a análise dos resultados sugere maior aprofundamento teórico, em especial dos aspectos educativos, do que os estudos internacionais.

Sobre o volume de produções sobre o tema, é possível que os estudos estrangeiros estejam em maior número que os nacionais, considerando a quantidade de periódicos 
específicos sobre o assunto e o volume de pesquisas. Além disso, existe um importante investimento de fundações e organizações, dentre outros espaços, que têm se dedicado a fomentar a indústria de jogos educativos, financiando, assim, tanto o seu desenvolvimento quanto estudos que possibilitem evidenciar sua eficácia educativa.

A avaliação da qualidade dos jogos digitais educativos não foi destacada como de importante interesse nas pesquisas. Assim como no Brasil, os estudos estrangeiros pouco têm investido neste tema, em particular para auxiliar os professores a selecionar jogos de qualidade para as práticas educativas. Quando esses estudos existem, tendem a se referir à qualidade na perspectiva do cliente ou no sentido de auxiliar os desenvolvedores de jogos digitais.

Um dado interessante identificado nos estudos estrangeiros foi a denominação dos jogos digitais para os cenários escolares como serious games. Com isso, parece existir, entre os pesquisadores, um entendimento de que os jogos como mediadores ou potencializadores de aprendizagem devem ser planejados desde a sua concepção. Neste contexto, parece não ser considerada, por exemplo, a identificação de elementos educativos em jogos que não foram planejados para esse fim, ou seja, os comerciais. No Brasil, de acordo com os dados levantados, não foi observado este tipo de compreensão. O que existe são jogos educativos, jogos comerciais de entretenimento utilizados em projetos escolares em plataformas interativas e jogos casuais também referidos nos espaços educativos, muito embora estas experiências sejam de caráter pontual.

\section{Conclusão}

Retomando as duas questões que nortearam este levantamento sobre "quais estudos propunham perspectivas para avaliar os jogos digitais com finalidade educativa?" e "quais avaliam a qualidade de jogos digitais com finalidade educativa?", foi possível concluir que se trata de um conhecimento em construção com o maior interesse da área de computação para investigação sobre o tema. $\mathrm{Na}$ área educacional, houve pequena contribuição nos últimos dez anos, em particular no Brasil. Não existe, até o momento, considerando os limites desta pesquisa, um instrumento que tenha por objetivo avaliar a qualidade dos jogos digitais para os cenários escolares de forma a permitir que o professor avalie a possibilidade de interação desses jogos no âmbito pedagógico. A preocupação com relação aos jogos e espaços escolares se apresenta, na maioria das vezes, como a necessidade de desenvolver serious games. O objetivo foca-se no desenvolvedor, sem considerar, por exemplo, o papel do docente como interator e sujeito deste processo. A qualidade dos jogos, quando investigada, sugere um caráter pontual e restrito. Mesmo que o estudo desenvolvido por (Savi, 2010) tivesse sugerido um modelo de avaliação com o interesse em avaliar a qualidade dos jogos, o autor não recomendou a possibilidade de sua replicabilidade em cenários escolares de forma genérica. A restrição foi apenas para o ensino da engenharia do software. E, mais ainda, o modelo de avaliação proposto por Savi não foi validado com jogos digitais, e sim com jogos analógicos. Kiili et al. (2014), que também focaram na avaliação da qualidade, tiveram como escopo auxiliar os games designers a melhorar o design do jogo para os fins educacionais e tomaram como referência a Teoria do Fluxo. 
Assim, considerando os dados revelados acima, valida-se a relevância de iniciativas que priorizem o campo da avaliação de jogos digitais, visando ampliar seu estudo na área de Educação. Tal atitude contribuirá para a integração dos jogos digitais na escola, na medida em que subsidiará a prática pedagógica através da participação ativa do professor. Além do que, iniciativas que invistam na área de Educação têm um papel fundamental em promover esta interlocução que, até então, parece se concentrar na indústria de jogos e nos seus desenvolvedores. Como trabalhos futuros, a necessidade de um framework avaliativo que priorize as questões educacionais voltadas a prática pedagógica, torna-se emergente.

a BNDES "Gedigames. " Relatório final - Mapeamento Brasileiro da Indústria brasileira e global dos jogos digitais", fev.2014.

${ }^{b}$ A exemplo de Squire (2004); Gee (2010), Alves (2012); Prensky (2012), Contreras-Espinosa, EguiaGomez e Hildebrand (2013); Moita et.al. (2013); Petry et. al. (2013); Coutinho e Alves (2016) dentre tantos.

${ }^{c}$ Este levantamento bibliográfico é parte da pesquisa de doutorado de uma das autoras que tem como objetivo avaliar a qualidade dos jogos digitais educativos através do desenvolvimento de um instrumento desenvolvido para este fim.

${ }^{d}$ Estas questões estão relacionadas aos objetivos do estudo.

\section{Referências bibliográficas}

AHMAD, M; RAHIM, L. A; ARSHAD, N. I. A review of educational games design frameworks: An analysis from software engineering. In: Computer and Information Sciences (ICCOINS), 2014 International Conference on. IEEE, 2014. p. 1-6.

AGUIAR, M.P. Jogos eletrônicos educativos: instrumento de avaliação focado nas fases iniciais do processo de design. 2010. 301 f. Dissertação (Mestre em Design). Programa de Pós-Graduação em Design do Setor de Humanas.

ALL, A.; CASTELLAR, E. P. N; LOOY, J. V. Measuring Effectiveness in Digital GameBased Learning: A Methodological Review. International Journal of Serious Games. Bruxelas. v.1, n.2, p.3-21.

ALVES L. Games, colaboração e aprendizagem. In: OKADA, A. (Org.). Recursos Educacionais Abertos e Redes Sociais: coaprendizagem e desenvolvimento profissional. Milton Keynes: Colearn, 2012, v. 1. p. 20-30.

ALVES L; FUENTES, L; JULIANO, M. Avaliação heurística como método potencial para avaliar a eficiência de um jogo educativo em transmitir seu conteúdo pedagógico. In: IX Seminário de Jogos Eletrônicos Comunicação e Educação, SJEECE, Salvador, 2013.p 1-9.

ARAÚJO, G.G. Um framework conceitual para apoiar para apoiar a instrumentação de avaliação formativa da aprendizagem em jogos digitais. 2013. 96.f. Dissertação (Mestre em Sistema da Computação). Programa de Pós-graduação em Ciência da Computação Universidade Federal do Rio Grande do Norte. Natal. 2013.

AZEVEDO, V.A. Jogos Eletrônicos e Educação. RENOTE. Revistas Novas Tecnologias na Educação. Porto Alegre. v.10. n. 3.p. 1-10. dez. 2012.

BELlOTI, F. et al. Assessment in and of serious games: an overview. Advances in Human-Computer Interaction, v. 2013, p. 1, 2013. 
BROCKMYER, J. H. et al. The development of the Game Engagement Questionnaire: A measure of engagement in video game-playing. Journal of Experimental Social Psychology, v. 45, n. 4, p. 624-634, 2009.

COUTINHO, I.J; ALVES, L.R. G. Os desafios e possibilidades de uma prática baseada em evidências com jogos digitais nos cenários educativos. In: ALVES, L.R.G; COUTINHO, I. J. (Org.). Jogos Digitais e Aprendizagem: fundamentos para uma prática baseada em evidências. Papirus, Campinas, 2016.p.105-122.

CONTRERAS- ESPINOSA, R.S; EGUIA-GÓMEZ, J.L. Pesquisa da avaliação e da eficácia da aprendizagem baseada em jogos digitais e reflexões em torno da literatura científica. In: COUTINHO, I.J. ALVES, L.R.G. (Org.). Jogos digitais e aprendizagem: fundamentos para uma prática baseada em evidências. Papirus, Campinas, 2016, p.61-76.

CONSALVO, M.; DUTTON, N. Game analysis: developing a methodological toolkit for the qualitative study of games. The Internacional Journal of Computer game research, v. 6, n. 1, 2006.p.1-17.

CSIKSZENTMIHALYI, M. Flow: the psychology of optimal experience. New York: Harper Perennial, 1990

DIAS, J.; ANDRADE, G.; SILVA, A.; HETKOWSKI, T. A Gênese híbrida de um Design: O Caso do Jogo/Simulador Kimera - Cidades Imaginárias. In: XI Simpósio Brasileiro de Games e Entretenimento Digital SBGames, São Paulo, Trilha da Computação, 2013.p.256-259.

DIAS, J, M. Procedimentos analíticos para avaliação de jogos educacionais digitais: uma experiência baseada no desenvolvimento do kimera. 2015. 226 f. Tese (Doutorado em Educação). Universidade do Estado da Bahia, Programa de Pós-Graduação em Educação. Salvador, 2015.

DE FREITAS, S.; OLIVER, M. How can exploratory learning with games and simulations within the curriculum be most effectively evaluated? Computer \& Education, v. 46, n. 3. p. 249-264. 2006

DOURADO, J. B, et al. Desenvolvimento e Avaliação de um Jogo com tecnologia de RA para auxiliar no ensino de matemática. In: XIV Simpósio Brasileiro de Games e Entretenimento Digital SBGames, Trilha da Cultura, Teresina, 2015.p. 846-853.

FRANÇA R. S.; TEDESCO, P. Explorando o pensamento computacional no ensino médio: do design à avaliação de jogos digitais

FU, F. L.; SU, R. C.; YU, S. C. EGameFlow: a scale to measure learners' enjoyment of e-learning games. In: Computer \& Education. Elsevier Educational Research Programme. 2009.

GEE, J.P. Bons Vídeos jogos + Boa aprendizagem: coletânea de Ensaios sobre os videojogos e Aprendizagem e a Literária. Portugal: Edições Pedágio, LDA. 2010.

GEE, D. et al. Assessing serious games: The GRAND assessment framework. Digital Studies/Le champ numérique, 2014.

GOMES, D. N. et.al. Ensino Aprendizagem através do Desenvolvimento de Jogos. In: XIV Simpósio Brasileiro de Games e Entretenimento Digital SBGames, Trilha da Cultura. Teresina. 2015. p.150-166. 
GURGEL, I. E. et.al. A Importância de Avaliar a Usabilidade dos Jogos: A Experiência do Virtual Team. In: I Simpósio Brasileiro de Games e Entretenimento Digital SBGames. Trilha de Art. e Design. Recife.2006. p. 1-9.

GRIMM G. H.; CALOMENO, C. Fundamentos para análise de Jogos Educacionais Digitais - aproximações da teoria semiótica e da mente representacional. In: Intercom Sociedade Brasileira de Estudos Interdisciplinares da Comunicação XXXII Congresso Brasileiro de Ciências da Comunicação. Curitiba, PR. 2009.p. 1-14.

HONG, J.-C. et al. Assessing the educational values of digital games. Journal of Computer Assisted Learning, v. 25, n. 5, p. 423-437, 2009.

JAPPUR, R.F. Modelo conceitual para criação, aplicação e avaliação de jogos educativos. 2014. 296.f. Tese. (Doutor em Engenharia e Gestão do Conhecimento). Programa de PósGraduação em Engenharia e Gestão do Conhecimento. Universidade Federal de Santa Catarina. Florianópolis. 2014.

JUNIOR, P.A.H; MENEZES, S. C. Modelo para um Framework Computacional para Avaliação Formativa da Aprendizagem em Jogos Digitais. In: XIV Simpósio Brasileiro de Games e Entretenimento Digital SBGames, Trilha da Cultura, Teresina, 2015. p. 819828.

KIILI, K., LAINEMA, T., DE FREITAS, S. and Arnab, S. Flow framework for analyzing the quality of Educational games. Entertainment Computing. v. 5. n. 4. p. 367-377. Agosto/ 2016.

LEITE, P.S; MENDONÇA, V. G. Diretrizes para Game Design de Jogos Educacionais. In: XII Simpósio Brasileiro de Games e Entretenimento Digital SBGames, Trilha de Art. e Design. São Paulo. 2013.p.132-141.

LEITE, P. S; JOSELLI, M. Wyz: Avaliação de um jogo mobile para auxílio no ensino da língua portuguesa às crianças com deficiência auditiva. In: XIV Simpósio Brasileiro de Games e Entretenimento Digital SBGames, Trilha de: Art \& Design, Teresina, 2015.p.483-50.

LEPE-SALAZAR, F. A Model to Analyze and Design Educational Games with Pedagogical Foundations. 2015.

MARCIANO, J. N.; MIRANDA, L. C.; MIRANDA, E. E.C. Evaluating multiple aspects of educational computer games: literature review and case study. International Journal of Computer Games Technology, v. 2014, p. 14, 2014.

MITGUTSCH K.; ALVARADO N. Purposeful by design? a serious game design assessment framework. Proceedings of the International Conference on the Foundations of Digital Games, 121-128. 2013.

MEDEIROS, M. D. O.; SCHIMIGUEL, J. Uma Abordagem para Avaliação de Jogos Educativos: Ênfase no Ensino Fundamental. In: Simpósio Brasileiro de Informática na educação (SBIE), 23, 2012, Rio de Janeiro. Anais. Disponível em: <http://www.brie.org>. Acesso em: 09.jul, 2016. p.1- 10.

MOHAMED, M. O.; JAAFAR, A. Challenges in the evaluation of educational computer games. In: INTERNATIONAL SYMPOSIUM ON INFORMATION TECHNOLOGY ITSIM, 4, 2010, Kuala Lumpur. IEEE, 2010, v. 1. p. 1-6.

MOITA, F.; LUCIANO, A.; COSTA, A; BARBOZA, W. Angry Birds como contexto digital educativo para ensino e aprendizagem de conceitos matemáticos: relato de um 
projeto. In: XII Simpósio Brasileiro de Games e Entretenimento digital SBGames, São Paulo, 2013.Trilha da Computação, 2013. p.121-127.

MORI, M. K.; PAUlinO, G. C.; MARTINS, F. E.; BATTAIOLA, A. L. Avaliação Heurística como Ferramenta para Levantamento de Requisitos na Produção de Games Educacionais. In: VII Simpósio Brasileiro de Games e Entretenimento, SBGames. Trilha de Computação, Belo Horizonte, 2008.

NACKE, L.; DRACHEN, A; GÖBEL, S. Methods for Evaluating Gameplay Experience in a Serious Gaming Context, International Journal for Computer Science and Sport Volume 9/Special Issue. p.1- 12.2010.

NEVES, D. E, et al. Avaliação de jogos sérios casuais usando o método Gameflow. Revista Brasileira de Computação Aplicada. Passo Fundo, v. 6, n. 1, p. 45-59, abr. 2014.

NESBIT, John; BELFER, Karen; LEACOCK, Tracey. Learning Object Review Instrument (LORI): user manual. [S. 1.], 2009. Disponível em: Acesso em: 3 out. 2016.

PETRY, A. et al. Parâmetros, estratégias e técnicas de análise de jogo: o caso. A mansão de Quelícera. In: XII Simpósio Brasileiro de Games e Entretenimento Digital SBGames. Trilha da Computação. São Paulo, 2013.p.141-151.

RIBEIRO, F.R. Jogos educacionais digitais para ensino de língua portuguesa: uma proposta de avaliação didático-pedagógica e ergonômica. 2013.136 f. Dissertação. (Mestre em Linguística Aplicada). Programa de Pós-graduação em Linguística Aplicada (PosLA). Centro de Humanidades da Universidade Estadual do Ceará. Fortaleza, 2013.

RODRIGUES, F. R. Instrumento para avaliação de jogos eletrônicos educativos do ensino fundamental 1. 2014.123 f. Dissertação. (Mestre em Linguística e Ensino). Programa de Pós-Graduação em Linguística e Ensino. Centro de Ciências Humanas, Letras e Artes da Universidade Federal da Paraíba. João Pessoa. 2014.

PINTO, M.; GOMEZ-CAMARERO, C.; FERNANDEZ-RAMOS, A. Los recursos educativos electrónicos: perspectivas y herramientas de evaluación. Perspectivas em Ciência da Informação, v. 17, n. 3, p. 82-99, 2012.

POURABDOLLAHIAN, B.; TAISCH, M.; KERGA, E. Serious games in manufacturing education: Evaluation of learners' engagement. Procedia Computer Science, v. 15, p. 256$265,2012$.

PRENSKY, M. Aprendizagem Baseada em Jogos Digitais. São Paulo: Senac, 2012.

ROCHA, R. V.; BITTENCOURT, I; ISOTANI, S. Avaliação de Jogos Sérios: questionário para auto avaliação e avaliação da reação do aprendiz. In: XIV Simpósio Brasileiro de Games e Entretenimento SBGames. Trilha de Art \& Design, Teresina, 2015.p.648-657.

SANDVIK, K. Evaluation of quality in computer games. Nordicom Review, n. 27, p. 267-283, 2006.

SAVI, R. Avaliação de Jogos voltados para disseminação do conhecimento. 2011.238.f. Tese. (Doutorado em Engenharia e Gestão de Conhecimento). Universidade Federal de Santa Catarina, Centro Tecnológico. Santa Catarina, 2011.

SENA A, C; MARZULO, L, A. J.; QUIRINO, W. F.; NASCIMENTO, A. P. Avaliação e Taxonomia de Jogos para Ensino de Programação de Computadores. In: XIV Simpósio Brasileiro de Games e Entretenimento SBGames. Trilha de Cultura, Teresina, 2015.p.776-785.

V. $14 \mathrm{~N}^{\mathrm{o}} 2$, dezembro, 2016 
SERRANO, Á. et al. A framework to improve evaluation in educational games. In: Global Engineering Education Conference (EDUCON), 2012 IEEE. IEEE, 2012. p. 1-8.

SILVA, P. F.; SILVA, T.P; SILVA, G. N. StudyLab: Construção e Avaliação de um aplicativo para auxiliar o Ensino de Química por professores da Educação Básica. Revista Tecnologias na Educação. Paraíba. Ano 7 - número 13. p. 1 -11, dez. 2015.

SWEETSER, P., \& WYETH, P. Gameflow: a model for evaluating player enjoyment in games. Computers in Entertainment, 3(3), 1-24. 2005.

SHCHIGLIK, C.; BARNES, S. J.; SCORNAVACCA JR, E. Developing Measures of Wireless Game Quality: A Three-Step Approach. In: ECIS. 2008. p. 2520-2531.

SQUIRE, K. D. Games, Learning and Society: Building a Field. Educational Technology. University of Wisconsin Madison. p. 51-54. 2007

OLIVEIRA, W. K, et al. Avaliação de Jogos Educativos: Uma Abordagem no Ensino de Matemática. In: Anais do XXVI Simpósio Brasileiro de Informática na Educação SBIE 2015. Maceió.2015. Anais do $26^{\circ}$ Simpósio Brasileiro de Informática na Educação SBIE 2015. Maceió: UFAL e IFAL, 2015.p 657-666.

TSUDA M., SANCHES V. M., FERREIRA T. G., OTSUKA J. L., BEDER D. M. Análise de métodos de avaliação de jogos educacionais. In: XIII Simpósio Brasileiro de Games e Entretenimento digital SBGames. Trilha de Art. \& Design. Porto Alegre,2015.

VALLE, P. H. D et al. HEDEG - Heurísticas para Avaliação de Jogos Educacionais Digitais.In: Nuevas Ideas en Informática Educativa TISE 2013.

VERIDIANO, D. A. S. Análise de jogos digitais para utilização no contexto escolar. Educ.\&Tecnol. Belo Horizonte v. 19. n. 3 p. 21-31 set. /dez. 2014 |

VICTAL, E.R.N; MENEZES.C.S. Avaliação para Aprendizagem baseada em Jogos: Proposta de um Framework. In: XIV Simpósio Brasileiro de Games e Entretenimento Digital SBGames, Trilha da Cultura, Teresina, 2015.p. 970-976.

VILARINHO, L.R.G; LEITE, M.P. Avaliação de jogos eletrônicos para uso na prática pedagógica: ultrapassando a escolha baseada no bom senso. RENOTE. Revistas Novas Tecnologias na Educação. Porto Alegre. v.1. n. 13. p.1-11. jul. 2015.

WIEBE, E. N. et al. Measuring engagement in video game-based environments: Investigation of the User Engagement Scale. Computers in Human Behavior, v. 32, p. 123-132, 2014. 